\title{
Digestión en horno de microondas para determinación de contenido de hierro y zinc totales en alimentos
}

\author{
Microwave digestion for determination of \\ iron and zinc content of total food
}

Paulina Silva Trejos

Fecha de recepción: 18 de octubre del 2011

Fecha de aprobación: 14 de abril del 2012

Silva, P. Digestión en horno de microondas para determinación de contenido de hierro y zinc totales en alimentos. Tecnología en Marcha. Vol. 25, No 3. Julio-Setiembre 2012. Pág 96-100. 


\section{Resumen}

En el presente estudio se optimizó el procedimiento de digestión de alimentos por medio de un horno de microondas, para cuantificar el hierro y el zinc totales en diferentes matrices por espectroscopía de absorción atómica. Se analizó la cantidad óptima de $\mathrm{HNO}_{3}$ concentrado al 65\% para digerir determinada masa de muestra por evaluación del porcentaje de recuperación obtenido con diferente cantidad de $\mathrm{HNO}_{3}$. Los resultados no difieren de los obtenidos por los métodos recomendados oficialmente de digestión ácida en sistemas abiertos y de calcinación.

\section{Palabras clave}

Digestión, microondas, mineralización, alimentos, hierro, zinc.

\section{Introducción}

En un gran número de ensayos en los cuales el objetivo es cuantificar la concentración total de diferentes minerales en matrices de alimentos, se requiere realizar una etapa previa de mineralización, con el fin de eliminar en su totalidad la materia orgánica y proceder a continuación con el análisis de la concentración del mineral.

El hierro y el zinc son micronutrientes que forman parte de proteínas y enzimas que actúan en diversos procesos biológicos indispensables para el funcionamiento de los seres vivos. Sin embargo, la dieta de la mayoría de las poblaciones es deficiente en estos micronutrientes. El hierro y el zinc se encuentran en una gran cantidad de alimentos, como frutas, verduras, cereales y leguminosas, pero sobre todo en carnes, pescados y mariscos (Valenzuela , 2008).

De todos los minerales, el hierro es el de mayor importancia biológica, debido a su influencia general sobre todos los procesos nutritivos de todos los tejidos, siendo su principal función transportar y ceder oxígeno. Existen resultados de la determinación de hierro en arroz, previa digestión de las muestras con $\mathrm{HNO}_{3}, \mathrm{HCl}$ y $\mathrm{HClO}_{4}$ en recipientes de teflón abiertos (Folch, 2007).

Los métodos tradicionales de mineralización de la materia orgánica para muestras de alimentos

\begin{abstract}
In this study, the procedure was optimized digestion of food by means of a microwave oven, to quantify the total iron and zinc in different matrices by atomic absorption spectroscopy. We analyzed the optimal amount of $65 \%$ concentrated $\mathrm{HNO} 3$ to digest sample mass determined by evaluating the percent recovery obtained with different amounts of $\mathrm{HNO}$. The results are similar to those obtained by the methods recommended officially acid digestion in open systems and calcination.
\end{abstract}

\section{Key words}

Digestion, microwave, mineralization, food, iron, zinc.

implican la digestión húmeda con mezclas de ácidos oxidantes, tales como el ácido nítrico, el ácido sulfúrico - mezclas de ambos en diferentes proporciones y, en uso limitado, también se recomienda el ácido perclórico. De acuerdo con los métodos oficiales de la AOAC (Association of Official Analytical Chemists), la mineralización de la materia orgánica se realiza por los métodos tradicionales de digestión húmeda y de calcinación.

Para la cuantificación de hierro y zinc por espectrometría de absorción atómica, se realiza la calcinación a temperaturas cercanas a $500^{\circ} \mathrm{C}$; la exactitud, expresada como porcentaje de recuperación, se reporta entre $97 \%$ y $106 \%$ para el hierro y entre 100\% y 106\% para el zinc en los diferentes grupos de matrices de alimentos (Universia, 20 I0).

En esta investigación se evaluó la exactitud en la cuantificación de hierro y zinc en diferentes clases de alimentos que forman parte de la dieta básica de los costarricenses.

Se propuso el método de digestión con horno de microondas debido a que, si bien es cierto el equipo utilizado es sustancialmente más caro, la manipulación de la muestra es mínima, evitándose la pérdida de analitos por volatilización, la contaminación de la muestra, la exposición por parte del analista a los vapores generados durante la digestión ácida y la 
disminución sustancial del tiempo invertido en la mineralización de la materia orgánica.

\section{Materiales y métodos}

Se utilizó un horno de microondas MilestoneEthos Plus para realizar la digestión de las muestras y un espectrómetro de absorción atómica Varian 220 Fastsequential, equipado con una lámpara multielemental para zinc y hierro.

Las disoluciones patrón utilizadas para obtener las curvas de calibración para cuantificar el hierro y el zinc se prepararon por dilución de disoluciones de $1000 \mathrm{mg} / \mathrm{L}$ en ambos minerales, trazables a la NIST®.

Los alimentos utilizados en esta investigación se trataron de acuerdo con la costumbre de ingesta de los costarricenses. Los que se consumen cocidos, se cocinaron con agua desionizada si agregarles ningún aditivo, grasa, aceite o sal, en horno de microondas hasta alcanzar la suavidad.

Una vez cocidos se liofilizaron y se midieron pares de muestras de igual masa en una balanza analítica, o alícuotas de igual volumen en el caso de alimentos líquidos; a una de las muestras pares no se le realizo adición y a la otra se le adicionó una alícuota de disolución patrón de cada mineral ensayado trazable a la NISTß.

Se usaron muestras de alimentos de productos lácteos (leche fluida, queso crema, queso Turrialba, queso mozzarella), pastas, huevos, salchichón, pescado, vainicas, carne molida, garbanzos, frijoles negros, arroz blanco, pan de bollito, pan cuadrado, zanahoria, plátano maduro, margarina, hígado de pollo, brócoli, cebolla, tortillas, pollo, lentejas, chile dulce, hígado de res y mondongo.

\section{Resultados}

En el cuadro I se presentan los parámetros obtenidos para las curvas de calibración obtenidas para cada mineral cuantificado en los alimentos y utilizadas para evaluar los porcentajes de recuperación.

En el ámbito de las concentraciones, se prepararon los patrones con concentraciones igualmente espaciadas y se obtuvo la curva de mejor ajuste por el método de mínimos cuadrados ponderados.

Para evaluar la cantidad óptima de ácido nítrico utilizado para digerir una masa fija de muestra seleccionada, de acuerdo con la capacidad máxima de muestra sólida o líquida recomendada por el fabricante del horno de microondas, se varió la cantidad adicionada de $\mathrm{HNO}_{3}$ al 65\%. Los datos reportados corresponden a la cantidad suficiente para digerir la totalidad de la muestra y a la que obtuvo el valor más cercano al I00\% de recuperación.

En los cuadros 2 y 3 se presentan los resultados de recuperación obtenidos para hierro y zinc, respectivamente, para muestras de masas y volúmenes de alimentos y la cantidad adicionada de ácido nítrico.

El programa utilizado para digerir fue el mismo para todas las muestras y se presenta en el cuadro 4.

\section{Discusión}

El método de digestión de muestras de alimentos en horno de microondas supera los métodos tradicionales de digestión ácida y calcinación. Los aspectos importantes por considerar son: el reducido tiempo utilizado para digerir cada muestra, la digestión se realiza utilizando únicamente ácido nítrico concentrado, y no mezclas de ácidos, se evita el contacto con vapores de ácidos como ocurre en la digestión ácida tradicional, las posibilidades de

Cuadro I. Condiciones de las curvas de calibración por espectroscopía de absorción atómica para hierro y zinc.

\begin{tabular}{|c|c|c|c|c|c|c|c|}
\hline Metal & $\begin{array}{c}\text { Ámbito } \\
\mathrm{mg} / \mathrm{L} \\
\text { patrones }\end{array}$ & $\begin{array}{c}\text { Número } \\
\text { fe }\end{array}$ & Pendiente & Intercepto & $\begin{array}{c}\text { Coeficiente } \\
\text { de } \\
\text { correlación }\end{array}$ & $\begin{array}{c}\text { LD } \\
\text { Meir\&Zund } \\
\mathrm{mg} / \mathrm{L}\end{array}$ & $\begin{array}{c}\text { LC } \\
\text { Meir\&Zund } \\
\mathrm{mg} / \mathrm{L}\end{array}$ \\
\hline Hierro & $\begin{array}{c}(0,0630- \\
1,50)\end{array}$ & 9 & 0,0600 & $0,0007 / 2$ & 0,9986 & 0,032 & 0,063 \\
\hline Zinc & $\begin{array}{c}(0,0350- \\
0,800)\end{array}$ & 9 & 0,460 & 0,00757 & 0,9985 & 0,018 & 0,035 \\
\hline
\end{tabular}


Cuadro 2. Porcentajes de recuperación de hierro obtenidos para la digestión de muestras de alimentos en horno de microondas con ácido nítrico concentrado.

\begin{tabular}{|c|c|c|c|}
\hline Alimento & Masa de alimento/g & $\mathbf{V} / \mathrm{mL} \mathrm{HNO}_{3} 65 \%$ & \% de recuperación \\
\hline Arroz blanco & 0,5 & 10 & 90 \\
\hline Brócoli & 1,0 & 20 & 110 \\
\hline Carne molida & 0,5 & 8,0 & 98 \\
\hline Cebolla & 1,0 & 20 & 84 \\
\hline Frijol negro & 0,5 & 8,0 & 100 \\
\hline Garbanzos & 0,5 & 8,0 & 98 \\
\hline Hígado de pollo & 0,5 & 8,0 & 99 \\
\hline Hígado de res & 1,5 & 9,0 & 106 \\
\hline Huevo & 0,5 & 6,0 & 110 \\
\hline Leche descremada & $5,0 \mathrm{~mL}$ & 9,0 & 103 \\
\hline Margarina & 0,5 & 8,0 & 99 \\
\hline Mondongo & 1,5 & 9,0 & 102 \\
\hline Pan bollito & 0,5 & 6,0 & 93 \\
\hline Pan cuadrado & 2,0 & 9,0 & 97 \\
\hline Pasta (tornillos) & 0,5 & 6,0 & 99 \\
\hline Pescado & 0,5 & 6,0 & 105 \\
\hline Plátano maduro & 0,5 & 10 & 90 \\
\hline Queso crema & 1,0 & 7,0 & 99 \\
\hline QuesoTurrialba & 1,0 & 8,0 & 103 \\
\hline Salchichón & 0,5 & 7,0 & 95 \\
\hline Tortillas & 0,5 & 6,0 & 96 \\
\hline Vainica & 0,5 & 5,0 & 90 \\
\hline Zanahoria & 0,5 & 10 & 900 \\
\hline
\end{tabular}

Cuadro 3. Porcentajes de recuperación de zinc obtenidos para la digestión de muestras de alimentos en horno de microondas con ácido nítrico concentrado.

\begin{tabular}{|c|c|c|c|}
\hline Alimento & Masa de alimento/g & $\mathrm{V} / \mathrm{mL} \mathrm{HNO}_{3}$ 65 \% & \% de recuperación \\
\hline Arroz blanco & 0,5 & 9,0 & 91 \\
\hline Carne molida & 0,5 & 9,0 & 105 \\
\hline Frijol negro & 0,5 & 10,0 & 92 \\
\hline Garbanzos & 0,5 & 10,0 & 91 \\
\hline Leche descremada & $5,0 \mathrm{~mL}$ & 9,0 & 103 \\
\hline Margarina & 0,5 & 8,0 & 99 \\
\hline Pan bollito & 0,5 & 9,0 & 98 \\
\hline Pescado & 0,5 & 7,0 & 85 \\
\hline Plátano maduro & 0,5 & 6,0 & 87 \\
\hline Queso crema & 1,0 & 9,0 & 90 \\
\hline Queso Turrialba & 1,0 & 8,0 & 103 \\
\hline Tortillas & 0,5 & 6,0 & 96 \\
\hline Zanahoria & 0,5 & 6,0 & 97 \\
\hline
\end{tabular}


Cuadro 4. Programa de digestión utilizado para el tratamiento de las muestras en horno de microondas.

\begin{tabular}{|c|c|c|}
\hline Etapa & Tiempo/minutos & Temperatura $/{ }^{\circ} \mathrm{C}$ \\
\hline Primera & 5 & 180 \\
\hline Segunda & 10 & 200 \\
\hline Tercera & 5 & 220 \\
\hline
\end{tabular}

pérdida del analito por volatilización no existen ya que la digestión se efectúa en recipientes de teflón cerrados herméticamente. La contaminación de las muestras no existe, debido a que se lleva a cabo en recipientes de teflón cerrados durante todo el proceso de digestión.

Los porcentajes de recuperación obtenidos para hierro oscilan entre $84 \%$ y $105 \%$ y para el zinc entre $85 \%$ y $105 \%$, para todas las clases de alimentos analizados.

Si se comparan los resultados de esta investigación con los obtenidos y reportados por métodos de mineralización tradicionales, no hay diferencia significativa (Universia, 2010). El carrusel del horno utilizado permitía la digestión simultánea de cinco muestras con control constante de las condiciones de temperatura y presión de las muestras durante el proceso.

Se recomienda la utilización de horno de microondas en lugar de los métodos de digestión con mezcla de ácidos en sistemas abiertos y calcinación, por las ventajas en tiempo, consumo de reactivos, peligrosidad y porcentajes de recuperación obtenidos. El alto costo del horno de microondas y los consumibles, comparado con el equipo utilizado en la digestión ácida y calcinación, se minimiza con los beneficios logrados y los excelentes resultados analíticos obtenidos (Silva, 2009a,b).

\section{Agradecimientos}

A la Vicerrectoría de Investigación de la Universidad de Costa Rica (UCR), por haber brindado los fondos; a la Escuela de Química de la UCR, por permitir el uso de los equipos de horno de microondas y el espectrómetro de absorción atómica, utilizados en la digestión y lectura de muestras respectivamente. De igual manera, agradece al Centro de Investigación en Tecnología de Alimentos (CITA) de la UCR, por la ayuda brindada para la liofilización de los alimentos utilizados como muestras en esta investigación.

\section{Bibliografía}

Valenzuela, C.; Letelier, M.A.; Olivares, M.; Arredondo, M. \& Pizarro, F. (2008). Determinación de hierro, zinc y cobre en carne de bovino. , Vol. 35, No. 2 [citado 201।-10-12], pp. 139-|46. Obtenido desde: http://www.scielo.cl/scielo. php?script=sci_arttext\&pid=S07 17

Folch, E.C. (2007). La determinación de hierro en arroz, , Vol. I0, No. 018 [citado 2011-10-12], pp. 129-137. Disponible en: http://redalyc.uaemex.mx/pdf/877/8770 I 809.pdf

Silva, P. (2009a). Validación de la metodología analítica para la determinación de hierro por espectroscopía de absorción atómica de llama en alimentos de la canasta básica costarricense. , Vol. 22, No. 2, Abril-Junio, pp. 20-22.

Silva, P. (2009b). Evaluación del contenido de hierro total en alimentos de la canasta básica de consumo de los costarricenses. ,Vol. 22, No. 2, Abril-Junio, pp.98-106.

Universia (20 I0). Manual de Análisis de Alimentos. Fundamentos y Técnicas. [citado 17/10/20II] Obtenido desde: http:// dspace.universia.net/bitstream/2024/1067// 